\title{
Dual Plane Augmentation Genioplasty Using Gore-Tex Chin Implants
}

Byung Jun Kim, Jong Woo Lim, ji Hoon Park, Yoon Ho Lee

Department of Plastic and Reconstructive Surgery, Seoul National University College of Medicine, Seoul, Korea

No potential conflict of interest relevant to this article was reported.
Background: The chin shape and position is important in determining the general shape of the face, and augmentation genioplasty is performed alone or in combination with other aesthetic procedures. However, augmentation genioplasty using osteotomy is an invasive and complex procedure with the potential to damage mentalis muscle and mental nerve, to affect chin growth, and prolonged recovery. Our aim was to present our experience with a modified augmentation genioplasty procedure for hypoplastic chins using a Gore-Tex implant.

Methods: Two vertical slit incisions were made at the canine level to create a supraperiosteal pocket between the incisions, preserving the periosteum and mentalis muscle. Minimal sub-periosteal dissection was performed lateral to the incisions along the mandibular border. The both wings of implant were inserted under the periosteum to achieve a stable dual plane implantation.

Results: In total, 47 patients underwent dual plane chin augmentation using a GoreTex implant between January 2008 and May 2013. The mean age at operation was 25.77 years (range, $15-55$ years). There were 3 cases of infection; one patient was treated with antibiotics, the others underwent implant removal. Additionally, two patients complained of postoperative parasthesia that spontaneously improved without any additional treatment. Most patients were satisfied with the postoperative outcomes, and no chin growth problems were observed among the younger patients.

Conclusion: Dual plane Gore-Tex chin augmentation is a minimally-invasive operation that is simple and safe. All implants yielded satisfactory results with no significant complications such as mental nerve injury, lower lip incompetence, or chin growth limitation.

Keywords: Chin / Genioplasty / Polytetrafluoroethylene / Bone growth

\section{INTRODUCTION}

The chin is located in the central portion of lower face and is a critical feature that contributes to the frontal profile and symmetry of the face. Microgenia is defined as an abnormally small chin. Unlike micrognathia, which designates hypoplastic mandibles with type II dental malocclusion, individuals with microgenia

\section{Correspondence: Yoon Ho Lee}

Department of Plastic and Reconstructive Surgery, Seoul National University College of Medicine, 101 Daehak-ro, Jongno-gu, Seoul 110-744, Korea E-mail: lyh2374@snu.ac.kr

*This article was presented at the 71th Congress of the Korean Society of Plastic and Reconstructive Surgeons on November 1-3, 2013.

Received June 30, 2014 / Revised July 25, 2014 / Accepted August 3, 2014 have normal dental occlusion. Microgenia is a cosmetic concern and contributes to the appearance of a disproportionately short face. A proportional nose can appear larger in comparison, and the neck soft tissue can sag due to the shortened fulcrum.

There have been many discussions on aesthetically pleasing profiles of the chin. Gonzalez-Ulloa and Stevens [1] described the ideal chin projection as a tangential line from the soft tissue nasion that is perpendicular to the Frankfort horizontal line. Farkas et al. [2] analyzed the inclination of the facial profile as an angle between the perpendicular line at the glabella and the line connecting the glabella and pogonion, which has been estimated to be around $-3^{\circ}$ in men and $-4^{\circ}$ in women. A few millimeters be- 
hind a perpendicular line on the lower lip in the Frankfort horizontal position has been widely accepted as a good reference for soft tissue pogonion [3].

Various treatment options have been developed to address chin deficiency. Although advancement genioplasty using osteotomy is the golden standard for chin augmentation, it is a complex procedure that requires general anesthesia and several days of hospitalization. Most patients prefer a simple, safe, and less invasive procedure. Autologous fat transfer or filler injection is a minimally invasive non-surgical option, but requires repeat treatments with unpredictable long-term outcomes.

Chin augmentation using alloplastic materials is a decent alternative to both osteotomies and fat/filler injections. The most commonly used materials include Silastic (solid silicone, Michigan Medical Co., Santa Barbara, CA, USA), Medpor (porous polyethylene, Porex Surgical Inc., College Park, GA, USA), and Gore-Tex (polytetrafluoroethylene, W.L. Gore \& Associates Inc., Flagstaff, AZ, USA). However, chin implantations have been associated with infection, migration, and mentalis muscle and nerve injury. Also, secondary bone resorption could injure tooth root or mental nerve and inhibit chin growth when the material is applied in patients while the mandible continues to grow.

We developed a new operative technique in which Gore-Tex chin implant is placed in two planes, with the intent to minimize aforementioned problems caused by alloplastic implantation. The aim of this study was to present our experience with the modified augmentation genioplasty procedure.

\section{METHODS}

This dual plane Gore-Tex chin augmentation procedure was performed on 47 patients between January 2008 and May 2013 by a single surgeon (L.Y.H). A retrospective review was conducted under the approval of the Institutional Review Board. Demographic data, type of anesthesia, operation time, combined surgery, and postoperative complications were analyzed. Subjective reports on the postoperative outcome were evaluated using the Global Aesthetic Improvement Scale (GAIS) (Table 1). Photographs taken preoperatively and postoperatively were evaluated by 2-blinded plastic surgeons not related to this study.

Most operations were performed under local anesthesia with intravenous sedation and analgesia. General anesthesia was administered to patients who were afraid to undergo the operation under local anesthesia, or who had undergone other surgeries such as face contouring surgery, rhinoplasty, or facelift procedure along with augmentation genioplasty. Surfaces were marked to indicate the area to be augmented, as well as the inferior mandibular border, canine, and mental foramen. Two regions for vertical bi-pedicle incisions of $1.5 \mathrm{~cm}$ in length were marked on the intraoral mucosa under the canine, $5 \mathrm{~mm}$ above the gingivobuccal sulcus. A mixture of 2\% lidocaine and 1:100,000 epinephrine was administered to the intraoral incision site and over the mental foramen. The intraoral mucosa was incised at two locations, and a supra-periosteal pocket was created under the mentalis muscle between the 2 incisions. A meticulous pocket dissection was performed parallel to the mentalis muscle to preserve as much of the muscle fibers as possible. The periosteal incision was made approximately $5 \mathrm{~mm}$ lateral to the mucosal incision in order to reduce the chance of infection. The periosteum was minimally elevated using a freer elevator along the inferior mandibular border up to the second premolar level.

The Gore-Tex chin implant used in this study had the following dimensions: width, $58 \mathrm{~mm}$; anteroposterior length, $44 \mathrm{~mm}$; height at midline, $15 \mathrm{~mm}$; and thickness at midline, $9.5 \mathrm{~mm}$, although implants of various sizes are commercially available. The Gore-Tex implant was sculpted to fit the contour of the mentum and lower border of the mandible. To prevent postoperative implant migration or protrusion, it is important to sculpt the implant such that it fits the mental protuberance and mental tubercle. The implant was inserted into the supra-periosteal pocket through the vertical mucosal incision after soaking it in betadine solution

Table 1. Global aesthetic improvement scale

\begin{tabular}{cl} 
Score & State \\
\hline 3 & Very much improved \\
2 & Much improved \\
1 & Improved \\
0 & No change \\
-1 & Worse \\
\hline
\end{tabular}


mixed with cefazolin to prevent infection. Through the opposite incision site, mosquito forceps were used to pull the implant from the incision on the other side. The midline of the implant was aligned to the symphysis, and the lateral wings of the implant were inserted into the sub-periosteal pocket to constrict freedom of movement. Depending on a patient's anatomy, the implant was trimmed to allow the lateral portions to fit the subperiosteal pocket. The distance between canines of lower jaw is known to be around $35.8 \mathrm{~mm}$ in adults [4]. We made periosteal incision at 5 $\mathrm{mm}$ lateral to the canine level, and 5 to $10 \mathrm{~mm}$ of implant could be inserted into the subperiosteal pocket on each side.

The wound was repaired in layers using 4-0 coated VICRYL (Ethicon, Somerville, NJ, USA). Externally, compressive dressing (Elatex, Alcare, Tokyo, Japan) was applied over implant location to prevent upward migration of implant which might occur with repeated mentalis contraction. The dressing was maintained for at least 2 weeks, until the implant was covered with soft tissue to prevent implant migration (Figs. 1, 2).

\section{RESULTS}

Among the 47 patients, 22 were male and 25 were female. Patient ages ranged from 15 to 55 years, with a mean age of 25.77 years. The mean follow-up period was 8.3 months (range, 1-29 months). Chin augmentation was performed alone in 21 patients, while 26 patients had undergone chin augmentation in conjunction with other procedures (augmentation rhinoplasty, 10; rejuvenation procedures such as face lift and fat grafting, 10; peri-alar augmentation, 3 ; other procedures, 3). Nineteen of the 21 patients had undergone isolated chin augmentation under local anesthesia, and the remaining two patients wished for general anesthesia to address the anxiety associated with conscious anesthesia. The mean operation time for chin augmentation alone was 43 minutes (range, 34-65 minutes). Three patients experienced postoperative infection. Antibiotic treatment was adequate in one patient, but the remaining two patients required implant removal. Reoperation was performed in 5 patients, mainly because of patient dissatisfaction regarding the size of the implants in the early postoperative period. Two patients complained of intermittent

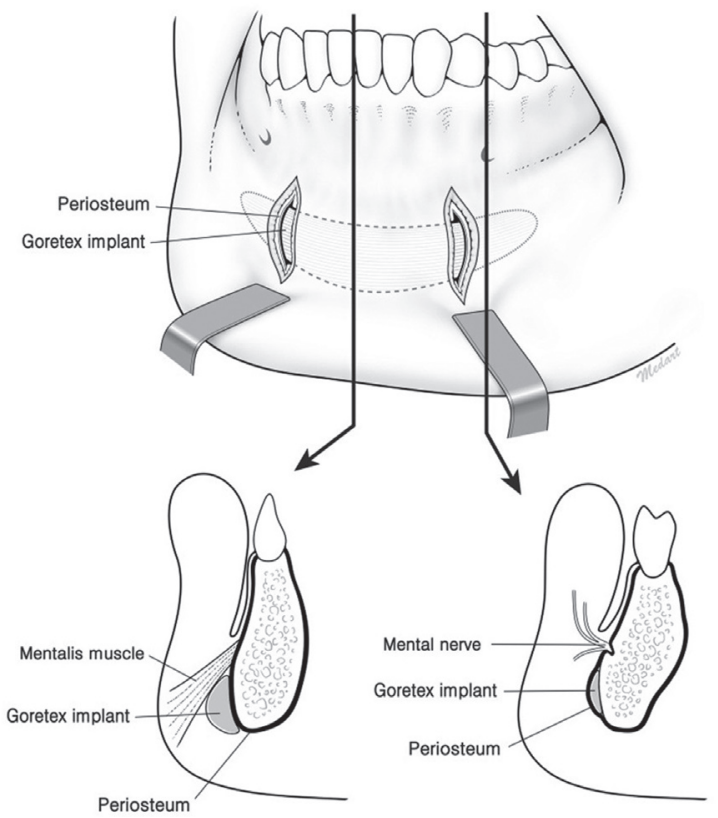

Fig. 1. Dual plane placement of the Gore-Tex implant. Stability can be achieved while preserving the mentalis muscle and minimizing sub-periosteal dissection.

parasthesia over the mental nerve distribution, but these symptoms resolved without any treatment within the first month. There was no incidence of mentalis muscle dysfunction, mental nerve injury, clinically obvious bony resorption, or bone growth retardation after surgery.

Postoperative GAIS was assessed at the last visit to the outpatient department, at least 1 month after surgery. The mean GAIS was 2.26, which represents satisfactory postoperative aesthetic results overall. Examples of photographs taken preoperatively and 6 month postoperatively are presented (Figs. 3, 4). Isolated augmentation genioplasty using Gore-Tex chin implant improved the profile and reduced soft tissue sagging of neck in lateral views. Furthermore, implant application contributed to vertical lengthening of the lower jaw in frontal views.

\section{DISCUSSION}

Expanded polytetrafluoroethylene (Gore-Tex, W.L. Gore \& Associates Inc.) has been used safely in facial plastic surgery for more than 20 years. Gore-Tex implants have micro-pores of $10-30 \mu \mathrm{m}$ on its surface, with negative charge on the surface. These proper- 

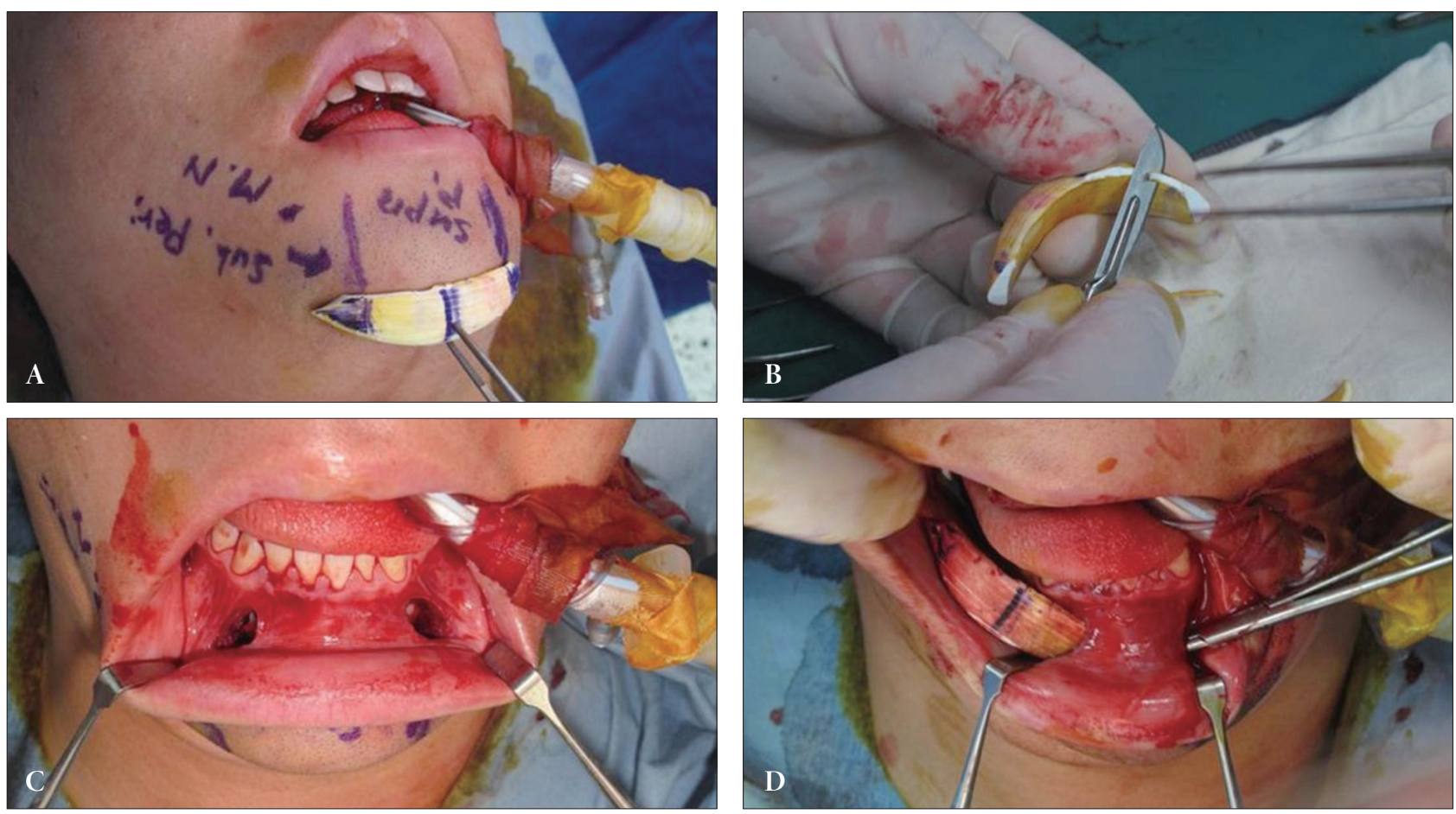

Fig. 2. Intraoperative photographs. (A) Midline and lateral portions of the subperiosteal placement marked on the Gore-Tex implant. (B) The Gore-Tex implant carved to fit the outline of the patient's mentum. (C) Two vertical bi-pedicle incisions made on the intraoral mucosa. (D) The implant inserted through one incision while mosquito forceps used to pull the implant from the other incision site.
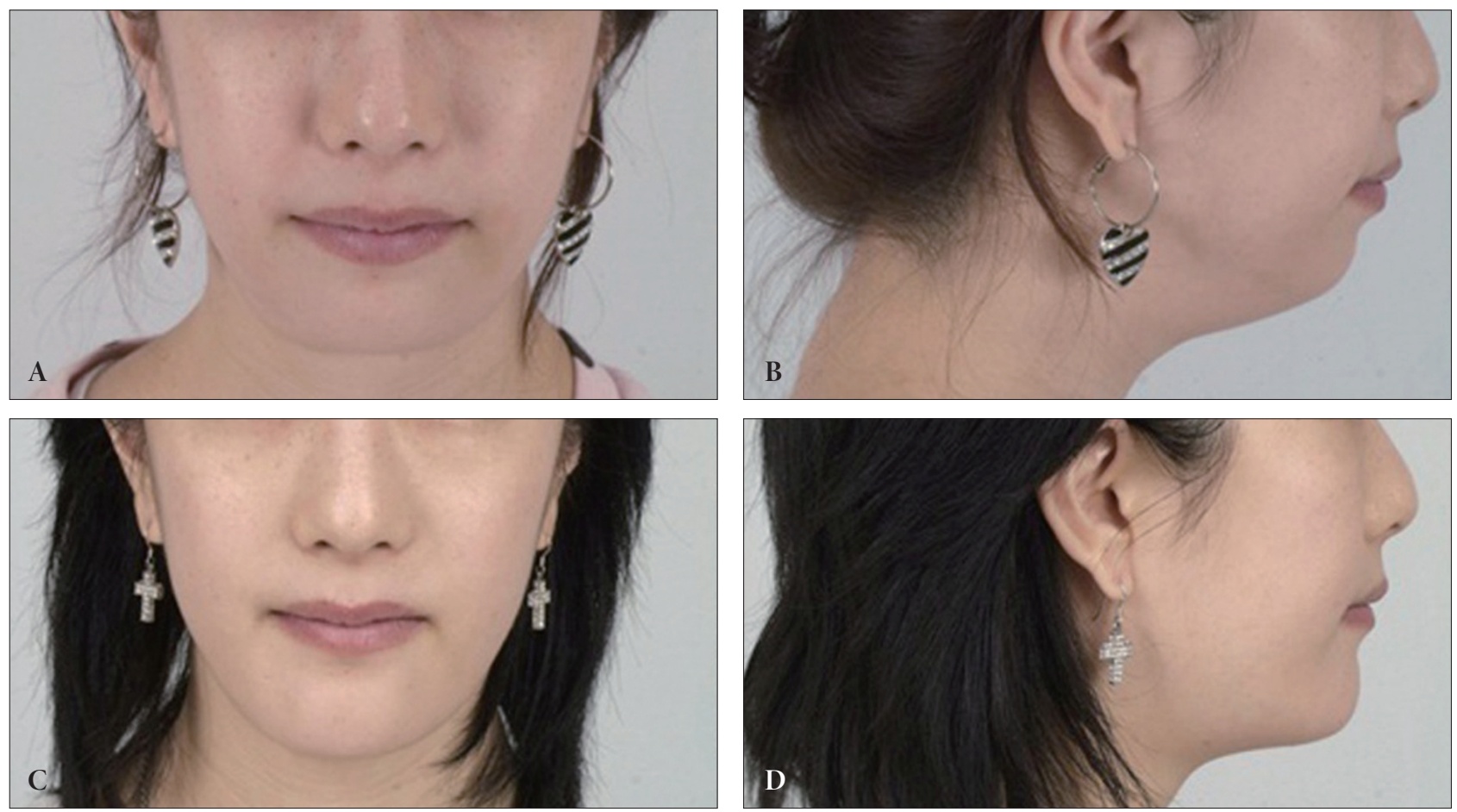

Fig. 3. A 24-year-old female patient who had undergone dual plane augmentation genioplasty with a Gore-Tex chin implant. Preoperative anteroposterior/lateral $(\mathrm{A}, \mathrm{B})$ and 6-month postoperative anteroposterior/lateral $(\mathrm{C}, \mathrm{D})$ photographs. 

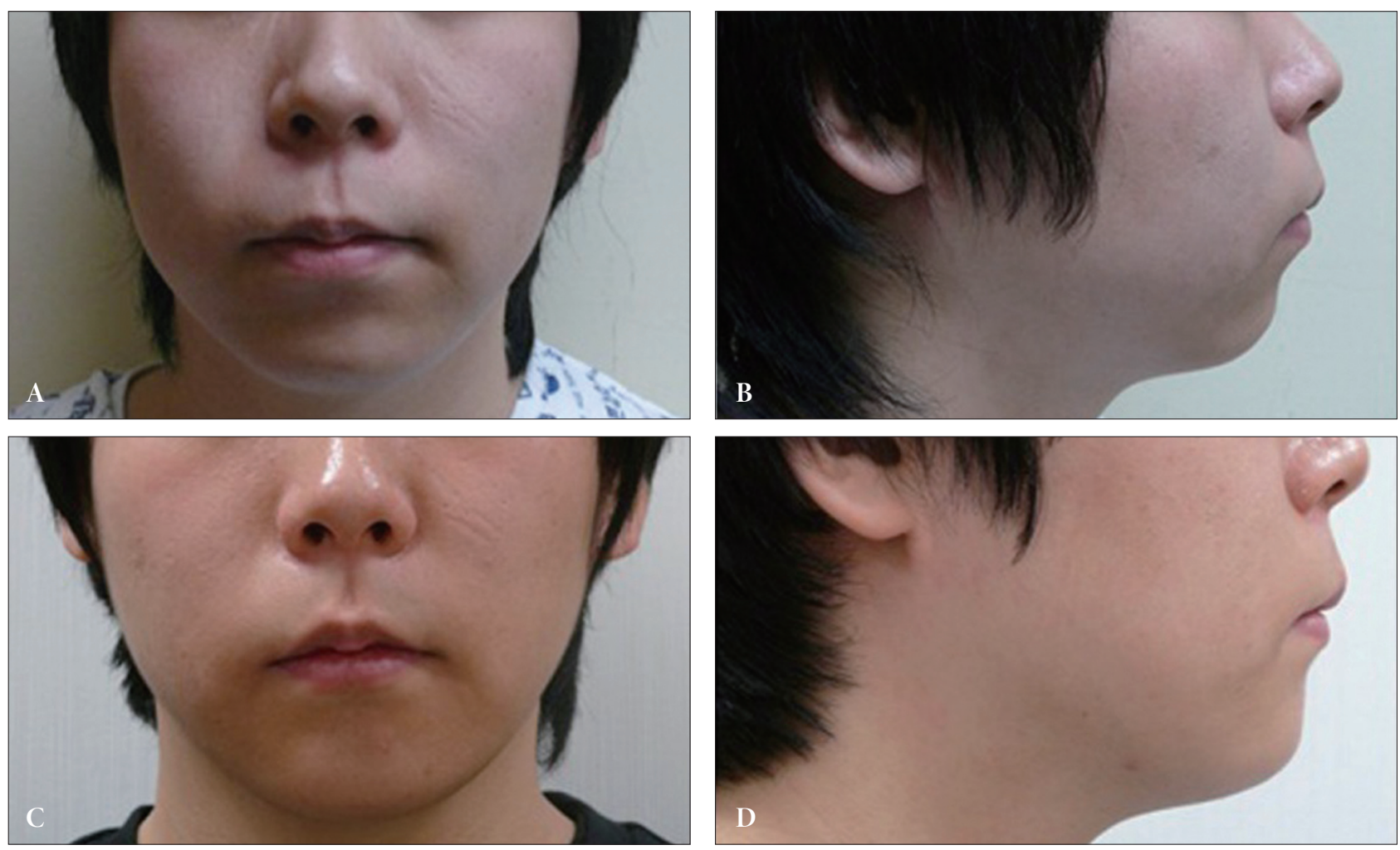

Fig. 4. A 20-year-old male patient who had undergone dual plane augmentation genioplasty with a Gore-Tex chin implant. Preoperative anteroposterior/lateral (A, B) and 6-month postoperative anteroposterior/lateral (C, D) photographs.

ties permit ingrowth of fibroblasts and capillaries that provide early stabilization of the implant with the surrounding soft tissue, while enabling easy removal when necessary. The porous surface of Gore-Tex enables supra-periosteal implantation, and capsule formation is less than that around silicone; therefore discomfort from mental nerve traction can be reduced. Furthermore, GoreTex is inert, non-carcinogenic, seldom allergenic, and causes minimal tissue reaction. In addition, Gore-Tex is pliable and could be easily trimmed and conformed to deep tissue bed.

Implantation of alloplastic materials has been contraindicated in children because of the possibility of bone growth retardation. It is generally accepted that facial osteotomies are not advised until growth completion (14-16 years old in females and 16-18 years old in males) [5]. Silicone implants inhibit bone growth when inserted under the periosteum, whereas bone growth is not interfered when implants are placed over the periosteum $[6,7]$. Two reasons have been suggested as a possible cause of bone resorption. One explanation is that implant pressure incites an inflam- matory reaction, which accelerates bone resorption. The other is that instability between the implant and bone surface could cause continuous inflammation which also inhibits bone growth. On the other hand, silicone implantation over the periosteum does not cause significant bone resorption. Therefore, intact periosteum and implant stabilization are important factors in bone growth inhibition. Progressive bone erosion can cause tooth root exposure and injure the mental nerve. The amount of bone erosion is associated with preoperative baseline labial incompetence and mentalis muscle hyperactivity [8]. The Gore-Tex implant can be inserted over the periosteum, and it can be used as an alternative to avoid bone erosion. A minimal amount of sub-periosteal dissection in our operative method causes minimal bone resorption and allows for the application of this procedure in adolescents without inhibiting growth potential.

However, Gore-Tex has some disadvantages, including a relatively high risk of local inflammatory reaction causing erythema and edema at the skin site, infection, and displacement. It also is 
relatively expensive. The overall infection rate of Gore-Tex in facial augmentation is reported to be around $1 \%[9,10]$. The presence of a sufficient amount of soft tissue around the implant, less tension, normal tissue without scarring, and the use of a proper implant size can reduce the chance of infection. The infection rate in the present study was $6.4 \%$, which is relatively high compared with previously published data, although statistical significance was not established because of the small sample size. It is thought that our intraoral approach increased the chance of contamination by normal flora when inserting the Gore-Tex implant. We made an effort to minimize the infection rate by adopting an aseptic procedure, irrigation of the implant with antibiotics and betadine, and proper selection of the recipient site and implant size. Suture fixation can be performed to prevent implant displacement; however, in our experience, accurate pocket formation and the use of a well-fitted implant along with postoperative taping are enough to prevent the implant migration.

There are concerns that it is difficult to maintain the volume and an exact shape of implant to provide structural support. Yoo et al. [11] investigated the volume change in Gore-Tex implants used in augmentation rhinoplasty with. They found a volume loss averaging $46.3 \%$ in $45 \times 4.0-\mathrm{mm}$ implants and $49.3 \%$ in $50 \times 5.0$ $\mathrm{mm}$ implants 4 to 26 months after rhinoplasty. However, the implants were generally inserted below the periosteum in patients who underwent rhinoplasty, whereas implants were placed in the supra-periosteal plane in our study. Furthermore, results obtained from a collection of problematic cases cannot be generalized. Jung et al. [12] evaluated the thickness of Gore-Tex using high-resolution ultrasonography, but could not find any significant changes over time. Godin et al. [13] reported their experience of 324 cases of chin augmentation using the Gore-Tex implant. No resorption or visible movement of any implant occurred within three years. These findings suggest that no significant changes in shape occur with Gore-Tex over time.

This technique does not damage the normal structure. Supraperiosteal plane dissection does not violate the mentalis muscle. The mentalis muscles are a pair of fan-shaped structures joined in the midline. They originate from the alveolar process of the mandible below incisors roots and have their insertion in the skin. The function of the mentalis muscles, which is to elevate the lower lip and chin, can be disrupted by a horizontal intraoral incision. Compared with the conventional horizontal incision, 2 vertical incisions minimize pain, bleeding, scar formation, tethering effects, and food becoming trapped between wound margins. In addition, every effort was made to keep the mental nerve undamaged. The position of mental foramen is below the second mandibular premolar root or in the interdental space between the first and second premolar teeth, approximately $1.5 \mathrm{~cm}$ above the mandible lower border. A region of at least $5 \mathrm{~mm}$ between the mental foramen and upper margin of the implant was preserved to decrease the risk of injury. An extraoral sub-mental approach can be a good alternative that prevents damage to the mentalis muscle and minimize infection from intraoral flora [3]. However, a noticeable scar is of great concern to cosmetic patients, especially among East Asian patients.

Dual plane Gore-Tex chin augmentation is a very simple method that takes less operative time. The average operation time was 43 minutes from incision to closure. Furthermore, local anesthesia with mental nerve block was sufficient for most cases of isolated augmentation genioplasty. However, it is difficult to correct asymmetric chin, vertical shortness, or complex deformity of the chin. Genioplasty with osteotomy could be an alternative in these cases, and ancillary correction could be performed using a GoreTex implant. The learning curve of this procedure lies in the creation of an adequate pocket size and preparing a Gore-Tex implant that conforms well to the underlying bone contour, which is important to prevent implant migration and exposure.

This study is limited by its retrospective design and small sample size. Furthermore, objective assessment tools should be performed to assess postoperative outcomes. The effect of the Gore-Tex chin implants on bone growth when placed over the periosteum should be investigated in future studies.

In conclusion, dual plane augmentation genioplasty using the Gore-Tex implant is an easy and effective surgical procedure that can produce aesthetically acceptable outcomes with minimal morbidity. Moreover, limited subperiosteal dissection preserves normal bone growth potential; therefore, it can also be used in younger patients who are still in their growth phase. 


\section{REFERENCES}

1. Gonzalez-Ulloa M, Stevens E. The role of chin correction in profileplasty. Plast Reconstr Surg 1968;41:477-86.

2. Farkas LG, Hreczko TA, Katic MJ. Craniofacial norms in North American Caucasians from birth (one year) to adulthood. In: Farkas LG, editor. Anthropometry of the head and face. New York: Raven Press; 1994. [Appendix A], p.241-335.

3. Yaremchuk MJ. Improving aesthetic outcomes after alloplastic chin augmentation. Plast Reconstr Surg 2003;112:1422-32.

4. Han MD, Jeon ES. The tooth size relationship between crowding patients and normal subjects in Korean. J Dent Hyg Sci 2006;6:29-34.

5. Freihofer HP, Bjork G, Jonsson E, Kuijpers-Jagtman AM. Timing of facial osteotomies. A consensus conference. Oral Surg Oral Med Oral Pathol 1994;78:432-6.

6. Arakaki M, YamashitaS, Mutaf M, Naito S, Fujii T. Onlay silicone and hydroxyapatite-tricalciumphosphate composite (HAP-TCP) blocks interfere with nasal bone growth in rabbits. Cleft Palate Craniofac J 1995;32:282-9.

7. Friedland JA, Coccaro PJ, Converse JM. Retrospective cephalometric analysis of mandibular bone absorption under silicone rubber chin implants. Plast Reconstr Surg 1976;57:144-51.

8. Matarasso A, Elias AC, Elias RL. Labial incompetence: a marker for progressive bone resorption in silastic chin augmentation. Plast Reconstr Surg 1996;98:1007-14.

9. Conrad K, Gillman G. A 6-year experience with the use of expanded polytetrafluoroethylene in rhinoplasty. Plast Reconstr Surg 1998;101:1675-83.

10. Godin MS, Waldman SR, Johnson CM Jr. The use of expanded polytetrafluoroethylene (Gore-Tex) in rhinoplasty. A 6-year experience. Arch Otolaryngol Head Neck Surg 1995;121:1131-6.

11. Yoo YC, Chung SI, Yang WY, Ko BM. Experience with use of expanded polytetrafluoroethylene(Gore-tex(R)) in cosmetic facial surgery. J Korean Soc Plast Reconstr Surg 2003;30:7-14.

12. Jung YG, Kim KH, Dhong HJ. Ultrasonographic monitoring of new expanded polytetrafluoroethylene implant thickness after augmentation rhinoplasty. Am J Rhinol Allergy 2012;26:e137-41.

13. Godin M, Costa L, Romo T, Truswell W, Wang T, Williams E. GoreTex chin implants: a review of 324 cases. Arch Facial Plast Surg 2003;5:224-7. 